\title{
Reducing Hospital Readmissions: A PatientBond Case Study
}

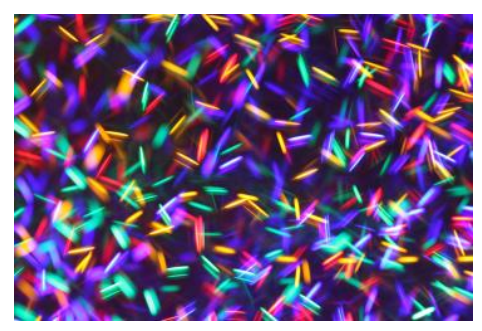

Brent Walker

Readmission penalties on hospitals hit a new high in 2016, increasing by a fifth over 2015 numbers to $\$ 528$ million. Yet, according to data from the Centers for Medicare \& Medicaid Services (CMS), the 30-day readmission rate is on the decline. Since the Hospital Readmission Reduction Program (HRRP) began in 2010, preventable readmissions have dropped by $8 \%$ nationally. ${ }^{1}$ So why will hospitals lose out on more than half a billion dollars in CMS reimbursements next year? Changes in how the rehospitalization rate is calculated influenced the jump in penalties. But that is not the only factor influencing 30-day readmissions. Patient engagement efforts often fall short, too.

\section{The Importance of Patient Engagement}

Preventable readmission penalties have encouraged hospitals to ramp up patient engagement efforts, but such penalties are not the only reason behind an increased focus on enlisting patients in their own care. To achieve meaningful healthcare reform, the Institute of Healthcare Improvement (IHI) has established the Triple Aim framework $(\text { Table } 1)^{2}$

Table 1. Steps to meaningful healthcare reform²

- Improve the patient experience in terms of quality and satisfaction

- Improve population health

- Reduce the per capita cost of healthcare 
Patient engagement is a critical component of the Triple Aim strategy, as well as efforts to activate the desired patient behaviors that reduce hospital readmissions. More than a decade ago, the University of Oregon developed a Patient Activation Measure (PAM) to better understand how individual healthcare consumers participate in managing their health. PatientBond Case Study: Reducing Hospital Readmissions health. Using the PAM measurement tool, which assesses patients' knowledge, skills and confidence related to healthcare, researchers found that low activation levels corresponded to nearly twice the risk of rehospitalization. ${ }^{3}$ The study's co-author and current research professor at the Health Policy Research Group at the University of Oregon, Judith Hibbard, suggests that, "Patient engagement is such a hot topic right now because we are all just recognizing that we aren't going to achieve better outcomes and lower costs unless patients are part of this, and they're able to do their part."

PAM, however, only tells part of the story. We have all seen physicians-who certainly have the required knowledge, skills and confidence to actively manage their health-fail to engage in healthy behaviors. Johns Hopkins' Schools of Public Health and Medicine reported that $53 \%$ of physicians are overweight or obese. ${ }^{4} \mathrm{~A}$ study published in the Journal of the American Medical Association found that a quarter of Licensed Practical Nurses smoke. ${ }^{5}$

If the most informed and educated people regarding health struggle to maintain healthy habits, how is the average patient expected to do any better? Obviously, education is an important part of driving healthy behavior change, but it is not the only answer. Education alone is not sufficient; it is critical that healthcare providers understand patient motivations, priorities and communication preferences in order to position education effectively.

Psychographic segmentation is a key to addressing these considerations. With psychographic segmentation, hospitals can crack the code to encouraging greater patient engagement. This type of segmentation delves deeper than demographics to identify differences in the motivations, beliefs and attitudes patients have towards health 
and wellness. It helps explain why patients do what they do, and how to appeal to their personal motivations to activate behavior change. In addition, it offers insights into where individuals turn for health advice and their communication preferences.

\section{Understanding How Patients Differ-Even with a Shared Diagnosis}

Patients with a given health condition no more think and act alike than do all members of an age group, gender or ethnicity. While they may share a common health condition, how they deal with it varies, and health is as much (if not more) an emotional consideration as a rational subject. Unless a healthcare provider can appeal to the patient's heart and mind, the desired behaviors may not occur, and the chances for a hospital readmission are heightened.

Psychographic segmentation empowers healthcare providers of all types to make more meaningful connections with patients. Rather than reaching out with one-size-fits-all communications, hospitals can leverage messaging that appeals to healthcare consumers on a deeper level based on their unique perspectives about health and wellness.

PatientBond, which automates patient communications (e.g., emails, text messages, Interactive Voice Response), leverages a proven psychographic segmentation model to customize messaging to each person's "health personality." This increases the likelihood of patient activation because engagement resonates more strongly.

This psychographic segmentation model was developed by healthcare consumer experts from Procter \& Gamble, who pioneered and refined such work during careers spanning several decades in P\&G's healthcare division. The model used by PatientBond balances $91.1 \%$ predictability with practical application using five distinct segments.

\section{Psychographic Segmentation Model}


The model identifies five segments of patients' health orientation: Balance Seekers, Willful Endurers, Priority Jugglers, Self Achievers, and Direction Taker (Figure 1)

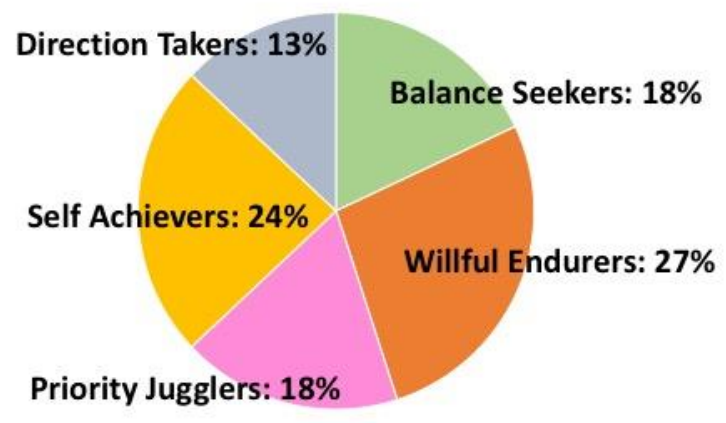

Figure 1. Four segments of patient health orientation

\section{Balance Seekers: $18 \%$ of the General Population}

Balance Seekers are generally proactive in their health and are wellness-oriented.

Balance Seekers are open to many ideas, sources of information, and treatment options when it comes to their healthcare. However, Balance Seekers themselves-not healthcare professionals-define what success looks like in their health. Physicians and other healthcare professionals are useful resources, but not the only resources, for leading a healthy life.

\section{Willful Endurers: $27 \%$ of the General Population}

Willful Endurers live in the "here and now" and believe there are more important things to focus on than improving their health for the future. Willful Endurers are not necessarily unhealthy, but they do what they like, when they like, and typically do not change their habits. They are self-reliant and can withstand anything life throws at them, going to the doctor only when they absolutely must.

\section{Priority Jugglers: $18 \%$ of the General Population}


Priority Jugglers are very busy with many responsibilities (e.g., career, family). Because they prioritize these responsibilities, Priority Jugglers may not take the time to invest in their own wellbeing, and they are reactive when it comes to health issues. However, Priority Jugglers are very proactive when it comes to their family's health and will make sure their loved ones receive the care they need.

\section{Self Achievers: $24 \%$ of the General Population}

Self Achievers are the most proactive when it comes to their wellness, investing what is necessary toward their health and appearance. Self Achievers may actually have health issues, but they stay on top of them with regular medical check-ups, health screenings, and research. Self Achievers are task oriented, and will tackle a challenge if they are given measurable goals.

\section{Direction Taker:13\% of the General Population}

Direction Takers believe their physician is the most credible resource for their healthcare needs. Direction Takers look to physicians and other healthcare professionals for direction and guidance because of their expertise and credentials. Direction Takers are more likely to go to the doctor at the first sign of health concerns. However, Direction Takers are not "Direction Followers" and may not always carry through on physicians' advice - not because they disagree with his/her recommendations, but because it is often difficult to work these recommendations into their routine.

Each psychographic segment has its own motivations and responds to unique messaging. What works with a Willful Endurer will not resonate with a Self Achiever. Effective patient engagement takes this into consideration in order to drive desired behavior change; using the same engagement approach with all five segments will face limited success.

While the percentages listed with each segment above represent the distribution of the segments across the General Population, the distribution will vary across sub- 
populations, such as age groups, ethnicities and people with a given health condition (Figure 2).

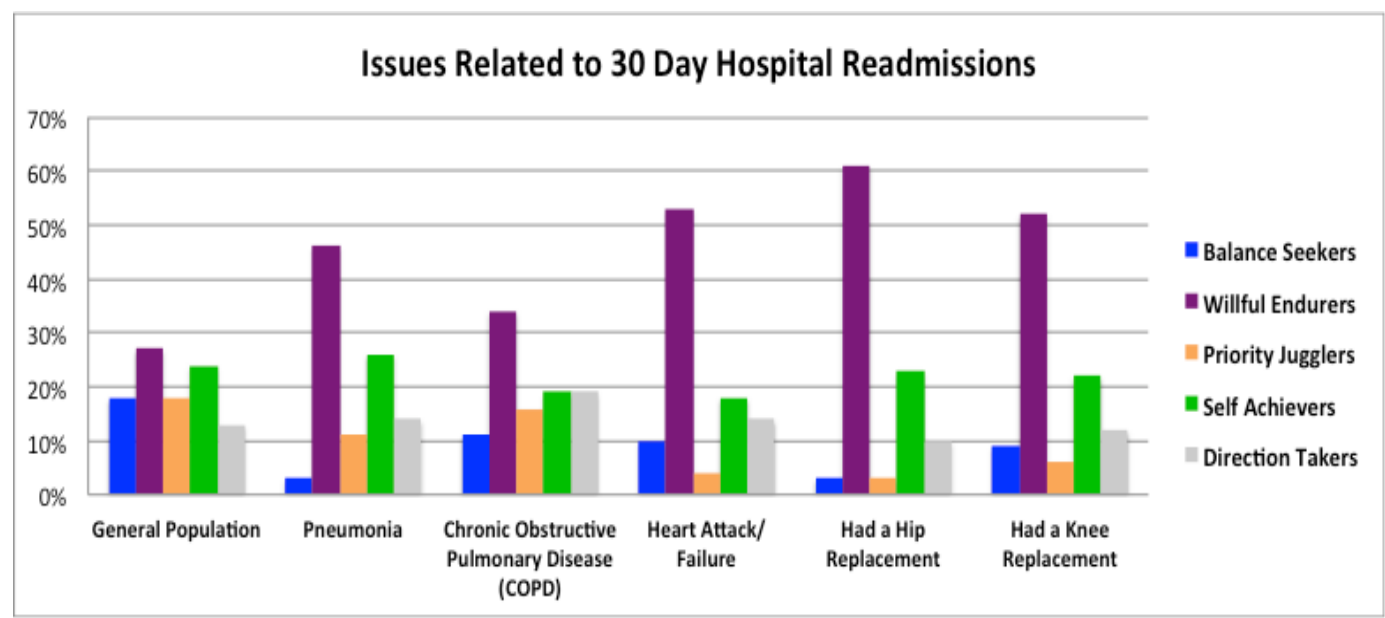

Figure 2. Segment distribution for health issues related to 30-day hospital readmissions tends to skew toward Willful Endurers

The over-development of Willful Endurers among issues related to hospital readmissions has profound implications. Willful Endurers are among the least likely to proactively manage their health condition, change habits that put them at greater risk or adhere to clinician instructions without the appropriate support.

Effective patient engagement with Willful Endurers requires an understanding of their motivations, priorities, and segment-specific key words and messages to influence their behaviors once they are discharged from the hospital.

\section{How Hospitals Can Lower Readmissions}

According to CMS estimates, one-fifth of Medicare patients return to the hospital within 30 days of discharge, and the Medicare Payment Advisory Commission suggests that $75 \%$ of those readmissions are preventable. ${ }^{3}$ Experts agree that hospitals must establish a strategy for reducing readmission rates (Table 2).

Table 2. A strategy for reducing readmission rates ${ }^{3}$ 
- Share patient discharge summaries with outpatient healthcare providers right away.
- This supports seamless transitional care which helps reduce readmissions.

- Ensure that patients and their family caregivers know whom to contact with questions or problems post discharge.

- Conduct a medication reconciliation to reduce medication-related readmissions.

- Implement an efficient follow-up process for discharged patients.

- Support a successful postdischarge transition with instruction and education.
- The CMS estimates that up to $11 \%$ of hospital readmissions are the result of medication non-adherence. ${ }^{6}$

- The key word here: Efficient. Your follow-up process needs to be manageable so that patients still in the hospital don't experience any gaps in their own care because nursing staff is juggling outreach to discharged patients as well.

- Better informed patients are more prepared to manage their health.

There are several transitional care programs that have been developed that reduce both healthcare costs and readmissions, including the Coleman model, the Naylor Model and Better Outcomes for Older Adults through Safe Transitions (BOOST) model. ${ }^{7}$ For example, Coleman's care transitions intervention model is a four-week program, which facilitates a smooth transition from the hospital or skilled nursing facility to the home.

Patient engagement is emphasized to address four pillars of readmission avoidance (Table 3).

Table 3. Four Pillars of Readmission Avoidance

- Medication self-management and compliance

- Maintenance of a personal health record

- Follow-up with a primary care physician

- Alertness to "red flags," such as symptoms or drug side effects, which increase the likelihood of readmission 
Each of these pillars relies on the patient's ability and willingness to act appropriately outside the walls of the hospital. This can be challenging to influence without an effective and efficient way of facilitating patient engagement remotely.

Leveraging an automated patient engagement platform enables hospitals to manage this type of process more time- and cost-effectively. And when it is supported by psychographic segmentation, hospitals can also ensure that they improve engagement among discharged patients by providing custom messages at the necessary cadence via the best channel.

\section{PATIENTBOND CASE STUDY: REDUCING READMISSIONS WITH AUTOMATED PATIENT ENGAGEMENT}

\section{The Challenge}

A prestigious New England hospital system wanted to reduce readmissions for a form of spine surgery (posterior lumbar spinal fusion), which had been sustained at $6 \%$. While $6 \%$ is a relatively low rate, each readmission costs up to $\$ 35,000$, so the prevention or reduction of readmissions was critical. The hospital department relied on three nurses working full time following up with patients to check on their post-surgery recovery status. Eventually, the patient load was such that the department requested a fourth nurse to address the ongoing challenge, but this was not feasible within the department's budget.

While the nurses were doing a yeoman's job tracking down patients and trying to engage them during the 30 days post-surgery, this process was resource intensive and was not lowering the readmission rate further. The chief of the hospital's orthopedic spine center needed to find a more efficient and effective way to reduce hospital readmissions.

\section{The Solution}

The hospital turned to PatientBond to establish a proactive, personalized patient engagement workflow for spinal surgery patients. The pilot program used a series of 
automated patient communications designed both to improve pre-op patient preparation and to help monitor patient progress and detect post-op recovery issues.

PatientBond built a white-labeled dashboard (Figure 3) for enrolling new patients for pre-op and post-discharge communications, and for monitoring patient response to these communications throughout the recovery process. During the pre-op consult one week prior to the procedure, patients answered a 12-question survey used to classify them into one of five psychographic segments.

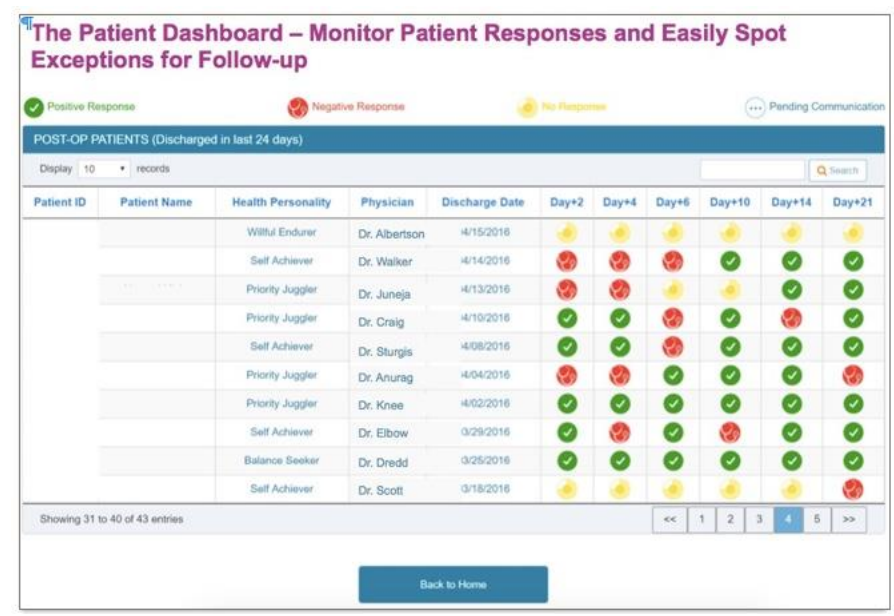

Figure 3. Patient Dashboard to monitor patient responses

Using this automated platform, the hospital then sent two communications: 5 days prior to surgery and 2 days prior to surgery, with education (e.g., a short video, links to articles) so patients could successfully prepare for their scheduled procedure. These communications - emails and text messages - featured customized content based on the patient's psychographic segment.

Initiating post-discharge communications was also easily accomplished-specifying the discharge date launched a series of custom instructional patient communications via email and text on days $2,4,6,10,14$, and 21 following the surgery. All communications included messaging designed to drive engagement based on a particular patient's 
psychographic profile and featured embedded patient actions—click and submit responses to recovery-related questions and links to additional education.

The dashboard allowed the department to focus on managing the exceptions-patients who provided negative or no response-to more efficiently allocate nursing resources to patients showing readmission risk indicators.

\section{Results}

Eight months into the pilot program, the hospital system has seen $85 \%$ engagement among patients to nine waves of communications, greater than $75 \%$ reduction in the time nurses spend on administration of the program and only one patient readmission. Clearly, the department has already seen a return on the investment. In addition to eliminating the need to add a nurse to the staff, the more efficient, automated process effectively freed up two nurses, enabling them to focus on in-patient needs. When combined with the savings associated with fewer readmissions, the pilot project has demonstrated a savings of $\$ 20$ for every $\$ 1$ invested.

Considering that the hospital also anticipates a boost in patient satisfaction, the use of the PatientBond platform has been a big win in driving patient engagement to reduce preventable 30-day readmissions.

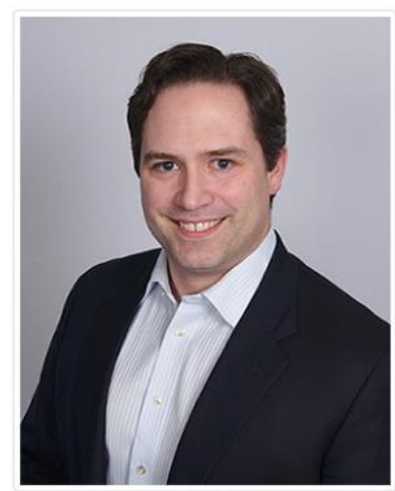

Brent Walker is EVP and Chief Marketing Officer for $c 2 b$ Solutions. Contact PatientBond for a demonstration of our capabilities: info@patientbond.com,www.patientbond.com,312445-8571.

References 
1. The CMS Blog. New data: 49 stated plus DC reduce avoidable hospital readmissions. 2019. URL: https://blog.cms.gov/2016/09/13/new-data-49-statesplus-dc-reduce-avoidable-hospital-readmissions/. Accessed 12/22/16.

2. Institute for Healthcare Improvement. The IHI Triple Aim. 2016. URL: http://www.ihi.org/engage/initiatives/tripleaim/pages/default.aspx. Accessed: 12/22/16.

3. H\&HN. Patient Activation Could be a Big Step Forward in Reducing Rehospitalizations. 2014. URL: http://www.hhnmag.com/articles/5291-patientactivation-could-be-a-big-step-forward-in-reducingrehospitalizations. Accessed 12/22/16.

4. Bleich SN, Bennett WL, Gudzune KA, Cooper LA. Impact of physician BMI on obesity care and beliefs. Obesity (Silver Spring). 2012 May;20(5):999-1005. URL: https://www.ncbi.nlm.nih.gov/pmc/articles/PMC3645927/. Accessed 12/22/16.

5. Sarna L, Bialous SA, Nandy K, Antonio AL, Yang Q. Changes in smoking prevalences among health care professionals from 2003 to 2010-2011. JAMA. 2014 Jan 8;311(2):197-9. URL: http://jamanetwork.com/journals/jama/fullarticle/1812944. Accessed12/22/16.

6. Shields B. Medication adherence strategies for lowering hospital readmission rtes. 2012. URL: http://www.mcknights.com/marketplace/medication-adherencestrategies-for-lowering-hospitalreadmission-rates/article/268557/\# edn3. Accessed 12/22/16.

7. Nelson JM, Pulley AL. Transitional care can reduce hospital readmissions. 2015. URL: http://www.medscape.com/viewarticle/844301 5. Accessed: 12/22/16.

Tags

PatientBond, how does telehealth enhance the doctor patient relationship, telehealth and telemedicine, telemedicine process improvement, telemedicine program implementation, telemedicine remote health, what is telemedicine and how does it work, reduce readmissions, hospital, patient engagement, Balance Seekers, Willful Endurers, Priority Jugglers, Self Achievers 